\title{
Remote spectral methods for detecting stress coniferous
}

\author{
Leonid Katkovsky ${ }^{1 *}$, Boris Beliaev ${ }^{1}$,Volha Siliuk ${ }^{1}$, Mikhail Beliaev $^{2}$, Erik Sarmin ${ }^{2}$, and \\ Yurii Davidovich ${ }^{1}$ \\ ${ }^{1}$ A.N. Sevchenko Institute of Applied Physical Problems of Belarusian State University, Minsk, \\ 220045, the Republic of Belarus \\ ${ }^{2}$ S.P. Korolev Rocket and Space Public Corporation Energia, Korolev, 141070, Russia
}

\begin{abstract}
The article presents investigation of the possibility of drying coniferous forest areas detecting by multispectral satellite data in the visible and NIR spectral range with low spatial resolution, obtained by the imaging systems of three satellites - the Belarusian spacecraft (BS), Landsat 8 and Sentinel 2. A forest area in the south of Belarus was considered as a test site. High-resolution multispectral airborne data and, in part, ground measurements were used as reference ground data by which training samples were formed. Most of the known classical methods of supervised classification have been tested, the maximum likelihood method turned out to be the best for this task. In order to improve the accuracy of identifying the drying areas of coniferous forests on multispectral images, parametric transformations of images in the spectral space are proposed, which should lead to an increase in initial small spectral differences. The methodological issues of assessing the accuracy of the satellite images classification are considered using the result of the classification of airborne image with high spatial resolution as a ground truth image. The assessment of the classification accuracy, both visually and using the obtained confusion matrices, allows us to conclude that the images of the BS, Landsat 8 and Sentinel 2 can be used to detect drying area of coniferous forests as well as the expediency of carrying out the proposed transformations of the original images.
\end{abstract}

\section{Introduction}

Sharp-dentated Bark Beetle (Ips acuminatus) has been recognized as one of the most dangerous pests in the EU countries, although it was previously considered an inactive species. Since 2010, a new pathological phenomenon has not been noted earlier on the territory of Belarus has been called "bark beetle drying of the pine". Extreme weather in recent years (decreased precipitation, lower water tables, drought and hurricane winds) which weakens trees is the main reason of pest outbreaks. Identification at early stages of damage to coniferous forests by various types of pests (bark beetle in the central and eastern part of Europe and silkworm in Siberian forests, other insects) is an urgent task of forestry.

\footnotetext{
* Corresponding author: katkovskyl@gmail.com
} 
Coniferous forests monitoring using satellite remote sensing [1,2] is preferable due to the wide coverage and the efficiency of obtaining up-to-date information [3]. There are some multispectral satellite sensors with a spatial resolution of 10-30 m, the data of which are in the public domain and usually cover large territories [4] than expensive images of very high $(1-10 \mathrm{~m})$ and ultra-high (less than $1 \mathrm{~m}$ ) resolutions. The interest of this study is finding out possibility of using such data for detecting of stress coniferous forest area.

The paper describes the methods and the results of the detection of drying forest area on satellite multispectral images, using reference samples (regions of interest in the images), which were visually detected on airborne very high spatial resolution images and then confirmed by ground measurements. The following remote sensing data were used: an airborne image obtained by a 4-band ADS-100 Leica camera from a height of several hundred meters (the spatial resolution during processing was roughened to $1 \mathrm{~m}$ ); a 4-band multispectral sensor image of the Belarusian spacecraft (BS) (spatial resolution $10.5 \mathrm{~m}$ ); the Landsat 8 OLI $(30 \mathrm{~m})$ and Sentinel $2(10 \mathrm{~m})$ satellite data (for both were taken 4 band lying in the visible and near-infrared spectral regions). All data were georeferenced. Airborne image, in contrast to the used satellite images, was in digital numbers (without radiometric correction, due to the lack of appropriate camera calibration). At first, satellite data were used for classification without atmospheric correction (in top-of-atmosphere radiance units), then - the same images after atmospheric correction (in bottom-of-atmosphere reflectance units).

In the first case (radiance images), in order to improve visualization and the possibility of making comparisons with the airborne image, pan-sharpening of the BS and Landsat 8 OLI (in the ENVI software package) was previously performed. After performing the supervised classification of the initial images, transformations of images in the spectral space were performed to enhance the spectral differences of objects (Section 2.1), which were also subjected to classification.

\section{Methods for the classification and detection of stress forest area on satellite images}

In the first step, small test region of forest contained stress area were chosen on satellite images (the test region was strictly coincided in all four images). The Landsat 8 image corresponding to the selected area contained only $16 \times 11$ pixels. In the second step, the selection of regions of interest (ROI) was made, which were used as training samples for each of the class. The classes were identified on visual interpretation of a high-resolution airborne image, confirmed by ground measurements, which was considered as a reference.

When comparing the classification results of images that differ greatly in spatial resolution (airborne and satellite), the problem of methodologically correct selection of ROI and the number of classes arises. For example, small-area "clean" ROIs in an airborne image may occupy only a small fraction of a pixel in a low-resolution satellite image, which is thus a mixture of different classes. At the same time, the choice of the same classes and their number is often problematic due to the difficulty of identifying some objects in low spatial resolution images. In particular, it is difficult to select the "tree shadow" class in a satellite image with a dense forest. One way to avoid this problem was to independently select the ROI on each image, as shown in Figure 1, where the ROIs are shown for the drying class in the airborne image as a background, and the composition of the classes was different in remote sensing images, but the drying class was present everywhere. 


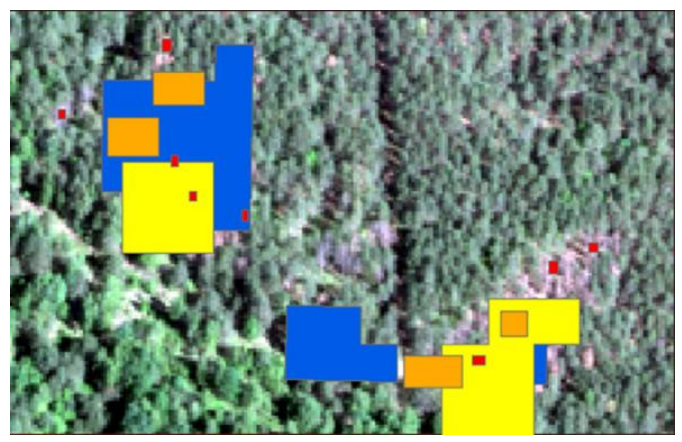

Fig. 1. ROI "stress" class, selected on airborne image (red), BS satellite image (orange), Sentinel 2 satellite image (blue) and Landsat 8 (yellow). The airborne image is shown as a background.

The next section 2.1 describes the proposed transformations that were applied to the original images.

\subsection{Spectral image transformations}

If we consider a multispectral image in $\mathrm{N}$-dimensional space with axes representing image bands, then it is represented in it as an N-dimensional "cloud" of points.

The greater the difference in brightness in the bands, the further away the points of the "cloud" are from the bisector of the N-facet angle formed by the coordinate planes. The transformation of a set of points, leading to their distance from the bisector, should lead, in our opinion, to an increase in the differences in the image bands, a decrease in their correlation, and an improvement in separability. The new N-bands image is calculated as:

$$
\bar{I}_{k, i j}=T_{i j} I_{k, i j}+(1 / N)\left(1-T_{i j}\right)\left(\sum_{n=1}^{N} I_{n, i j}\right)
$$

Here $I_{n, i j}, \bar{I}_{k, i j}$ - original and transformed images respectively, $k, n$ - bands numbers, $(i, j)$ - pixel number (row and column). The image parameter sets the degree of amplification of the initial interband differences of the image and depends on the distance $d_{i j}$ of the current pixel $(i, j)$ to the bisector of the $\mathrm{N}$-facet angle:

$$
d_{i j}=\sqrt{\frac{1}{N} \sum_{n>m=1}^{N}\left(I_{m, i j}-I_{n, i j}\right)^{2}}
$$

Two options are proposed for $T$. In the first option $T_{i j}$ is inversely proportional to $d_{i j}$ :

$$
T_{i j}\left(d_{i j}\right)=\left(T_{\max } d_{\max }\right) /\left\{d_{\max }+d_{i j}\left[\left(T_{\max } / T_{\min }\right)-1\right]\right\}
$$

Here $d_{\max }$ - maximum value $d_{i j}, T_{\max }, T_{\min }$ - set conversion parameters, respectively, maximum and minimum values of $T_{i j}$. It is easy to show that the deviation of the point of the new multispectral image from the bisector is $\bar{d}_{i j}=T_{i j} d_{i}$. From formula (3) it follows that $\operatorname{Tij}\left(d_{\max }\right)=\operatorname{Tmin}$ and $T_{i j}(0)=T_{\max }$ (if there are points lying on the bisector), i.e. points lying closer to the bisector move away from it as much as possible, and those lying further away minimum [5].

As a second option, it is proposed to use a directly proportional relationship $T_{i j}\left(d_{i j}\right)$ from $d_{i j}$ of the following kind:

$$
T_{i j}\left(d_{i j}\right)=T_{\min }\left[1+\left(d_{i j} / d_{\max }\right)\left(T_{\max } / T_{\min }-1\right)\right]
$$

Wherein $T_{i j}\left(d_{\max }\right)=T_{\max }$ and $T_{i j}(0)=T_{\min }$, i. e. points farther from the bisector are removed farther than closer ones. With regard to a multispectral image, relatively small 
differences in the bands (points lying near the bisector) do not make sense to significantly enhance, since these differences will usually be caused by measurement errors (noise). At the same time, noticeable differences, even more enhanced, should give better discrimination of objects, based precisely on the values of the brightness in individual bands. The values of the transformation parameters (3), (4), $T_{\max }$ and $T_{\min }$ are selected empirically by trial calculations. Image transformation and classification were performed in the ENVI program.

\subsection{Classification results}

When classifying, on the image covers a small area of forest four classes were set: stress, healthy coniferous forest, deciduous forest and shadows. The degree of separability of the specified classes was estimated by the selected ROI using two parameters: the JeffreysMatusit separability measure and the transformed divergence, the values of which (close to 2) showed very good separability every time. However, a certain methodological problem arises when assessing the classification accuracy of satellite images. If we evaluate it in relation to the selected ROI (ground truth ROI), then it was high in all cases (more than $90 \%$ ) and is not presented here.

Since the classification accuracy of the original airborne image of the ADS camera was very close to $100 \%$ for all classes (according to ROI), it was logical to use the classified ADS image as the ground truth image. For this purpose, in order to compare the classification results, it was assumed that all ROIs should be the same on all images (of different resolutions), while their position and size are determined by geographic coordinates.

Thus, the results of the classification from the airborne images were used as a reference. To construct a confusion matrix for all satellite images from an ADS airborne image as a ground truth image, it was necessary to convert the satellite images to ADS resolution $(1 \mathrm{~m})$ and the same number of pixels (Layer stacking operation in ENVI). Finally, it is advisable to group neighboring pixels (clump classes) in a high-resolution classified image (in order to bring the resolutions of the compared images closer together), which often improves the classification accuracy, which is given by the reference image error matrix (ADS).

Examples of the results of image classification are presented in Table 1, where the second column shows the original images, and the images of the BS, Sentinel 2, Landsat 8 OLI are resampled to the resolution of the ADS aerial image. In the next column - the results of classification by the maximum likelihood method - the "drying" class is overlayed on the original images, and before classification one of the transformation options in the spectral space was carried out (formulas (1) - (4)). The last column of the table shows the classification accuracy: general and by the drying class to classify the original image and the best version of the converted image, for ADS the accuracy is given in relation to the original ROI, for satellite images - according to the error matrix, where the ground-based true data is the ADS classification.

At the second stage of the classification, the same images, but in reflectance units (after atmospheric correction, except for ADS) were taken. But the area of forest was much larger (the Landsat image already contained 112x52 pixels), the scene contained a greater number of classes (non-forest objects in Fig. 2 are masked in black)), seven classes were already defined for the forest instead of four.

Figure 3 shows the classification results for ADS and BS (overlaying the drying class on the converted original images. 
Table 1. Results of determining forest stress from transformed multispectral images by the maximum likelihood method.

\begin{tabular}{|c|c|c|c|}
\hline $\begin{array}{c}\text { Imaging } \\
\text { system }\end{array}$ & $\begin{array}{l}\text { Original images, } \\
\text { satellite - after stretching to } \\
\text { ADS resolution }\end{array}$ & $\begin{array}{c}\text { ADS and satellite } \\
\text { classification results } \\
\text { converted by one of the } \\
\text { options }\end{array}$ & $\begin{array}{c}\text { Accuracy of } \\
\text { classification, } \\
\text { total / according to the } \\
\text { drying class according } \\
\text { to the original / } \\
\text { according to the } \\
\text { transformed, } \%\end{array}$ \\
\hline ADS & 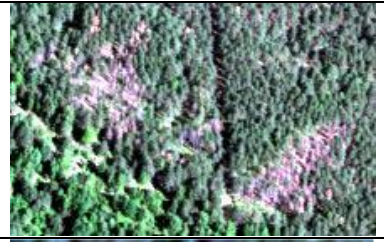 & 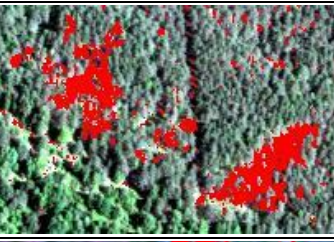 & $99,6 / 100$ \\
\hline BS & & & $\begin{array}{l}51,6 \text { / 57,8 / 60,9 } \\
\text { After clump classes by } \\
\text { 7x7 pixels: } \\
\text { 96,8/85,6 }\end{array}$ \\
\hline Sentinel 2 & & & 52,5 / 56,5 / 57,5 \\
\hline $\begin{array}{c}\text { Landsat } 8 \\
\text { OLI }\end{array}$ & & & $55,7 / 56,8 / 57,2$ \\
\hline
\end{tabular}

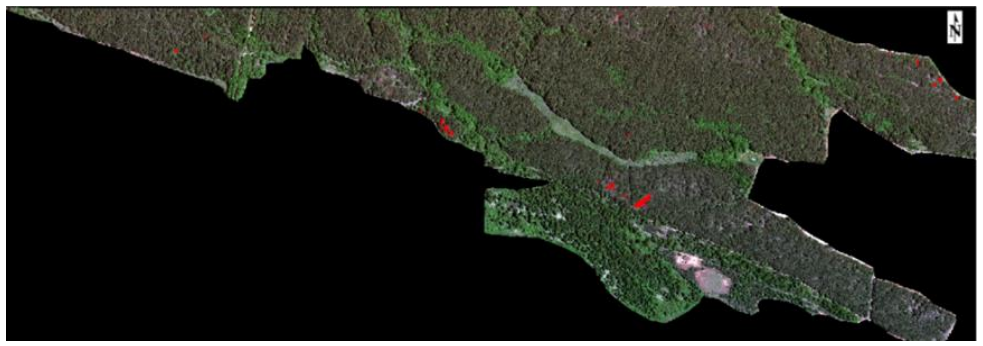

Fig. 2. Overlay ROI “drying” highlighted in airborne image (red)

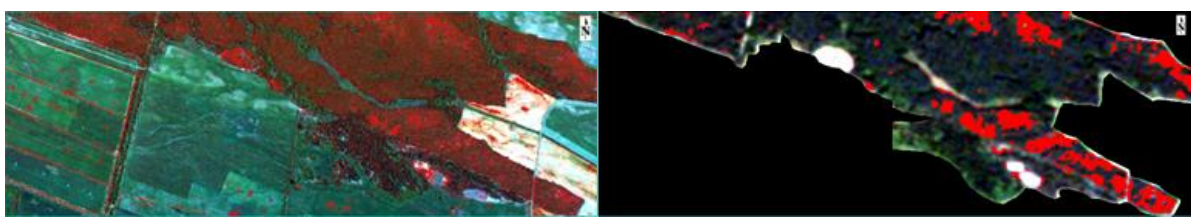

Fig. 3. Overlay of the "drying" class (red) on the images of ADS (left) and BS (right) 
The classification accuracy of the ADS image relative to the target ROI was $99.8 \%$ (overall) and $81.9 \%$ for the "drying" class. For satellite imagery, the accuracy relative to the ADS classification of the original and converted imagery is shown in Table 2.

Table 2. Accuracy of satellite imagery classification (original and transformed) relative to ADS classification

\begin{tabular}{|c|c|c|}
\hline Imaging system & $\begin{array}{c}\text { Classification } \\
\text { accuracy, total/drying, } \\
\text { \% (original images) }\end{array}$ & $\begin{array}{c}\text { Accuracy of } \\
\text { classification, } \\
\text { total / drying, \% } \\
\text { (converted images) }\end{array}$ \\
\hline BS & $94,1 / 17,1$ & $94,5 / 24,6$ \\
\hline Sentinel 2 & $95,3 / 14,5$ & $97,4 / 16,5$ \\
\hline Landsat 8 OLI & $94,3 / 24,5$ & $94,4 / 23,3$ \\
\hline
\end{tabular}

\section{Conclusion}

The obtained classification results allow us to conclude that the images of the BS, Landsat 8 and Sentinel 2 can be used for reliable detection of drying several tens of meters areas of coniferous forests, while it is advisable to carry out the proposed transformations of images in the spectral space, which enhance their discrimination. The benefits of using images after atmospheric correction were not clearly identified, but this may be due to different image sizes before and after correction, and to resampling (strong smoothing).

\section{References}

1. H. Abdullah, R. Darvishzadeh, A.K. Skidmore, M. Heurich, Remote Sens. 11 (2019).

2. V.I. Kharuk, S.T. Im, M.L. Dvinskaya, Russian Journal of Ecology, 3 (2016).

3. I.A. Saigin, S.A. Bartalev, F.V. Stytsenko, A method for detecting long-term drying out of dark coniferous forests in Russia based on satellite data, in Proceedings of the 17th All-Russian Open Conference "Current Problems of Remote Sensing of The Earth From Space”, Space Research Institute, 11-15 November 2019, Moscow, Russia (2019).

4. Yu.S. Davidovich, L.V. Katkovsky, Recognition of tree vegetation stress in aerospace imagery, in Proceedings of the GIS technologies in geosciences, BSU, 13 November 2019, Minsk, Belarus (2019).

5. B.I. Belyaev, L.V. Katkovskiy, Optical remote sensing, BSU, (2006). 